\title{
On Some Approximately Balanced Combinatorial Cooperative Games
}

\author{
ULRICH FAIGLE AND WALTER KERN \\ Department of Applied Mathematics, University of Twente, 7500 AE Enschede, The Netherlands
}

\begin{abstract}
A model of taxation for cooperative $n$-person games is introduced where proper coalitions are taxed proportionally to their value. Games with non-empty core under taxation at rate $\varepsilon$ are $\varepsilon$-balanced. Sharp bounds on $\varepsilon$ in matching games on (not necessarily bipartite) graphs are established. Upper and lower bounds on the smallest $\varepsilon$ in bin packing games are derived and euclidean random TSP games are seen to be, with high probability, $\varepsilon$-balanced for $\varepsilon \approx 0.06$.
\end{abstract}

Key Words: cooperative game, balanced, core, tax, matching, bin packing, traveling salesman

\section{Introduction}

For our purposes, a cooperative game is just a function $v$, the characteristic function of the game, that assigns to each coalition $S \subseteq N$ of a finite set $N$ of players a real number $v(S)$. We consider games where $v(S)$ arises as the optimal value of a combinatorial optimization problem defined by $S$. Typically, "optimization" will mean "maximization" (e.g., matching, bin packing). In case of "minimization", we speak of a cost game (e.g., minimum spanning tree, traveling salesman). The discussion of minimization games is completely analogous to maximization games.

A solution concept tries to distribute the value $v(N)$ of the grand coalition $N$ among the individual players in an acceptable manner. Doubtless, the intuitively most attractive solution concept is the core of a game, which consists of those vectors $x \in \mathbb{R}^{N}$ with component sum $x(N)=v(N)$ and $S$-restricted component sum $x(S) \geq v(S)$ for all $S \subset N$. Games with a non-empty core are balanced.

Unfortunately, many interesting games are not balanced. This means that one either has to introduce a completely different solution concept (e.g., Shapley value) or one has to modify the notion of "core". A model for the latter goes back to Shapley and Shubik [1966]. In that model, a proper coalition $S \subset N$ is taxed by a constant $\varepsilon$ or with rate $\varepsilon$ proportionally to its size so that the value is reduced to $v(S)-\varepsilon$ or $v(S)-\varepsilon|S|$ resp. . Tijs and Driessen [1986] propose a tax proportional to the difference $v(S)-\sum_{i \in S} v(i)$. One now seeks a core vector in the taxed game, thereby trying to keep the tax(rate) $\varepsilon$ "small". 
In the present note, we suggest a model of taxation where the tax imposed on a proper coalition $S \subset N$ is proportional to its value $v(S)$. This is the usual idea behind a sales tax and, therefore, appears to be quite realistic. We thus reduce that value $v(S)$ to $(1-\varepsilon) v(S)$. A game with non-empty core after taxation with rate $\varepsilon$ is $\varepsilon$-balanced. In this sense, $\varepsilon$-taxation provides an $\varepsilon$-approximation to balancedness.

In Section 2, we relate $\varepsilon$-balancedness to the usual definition of balancedness via the analogue of Bondareva's [1963] and Shapley's [1967] theorem. The optimal $\varepsilon$ for matching games on arbitrary (possibly non-bipartite) graphs is determined in Section 3. Section 4 introduces bin packing games and derives (not sharp) bounds on the best $\varepsilon$. Finally, we discuss traveling salesman games and argue that large euclidean game instances are, with high probability, $\varepsilon$-balanced for $\varepsilon \approx 0.06$.

\section{$2 \varepsilon$-Balancedness and $\varepsilon$-Core Allocations}

In this section, we introduce the notion of $\varepsilon$-balanced games and the $\varepsilon$-core of a game. Arguing along the same lines as in Bondareva [1963] and Shapley [1967], we conclude that $\varepsilon$-balanced games are characterized by the existence of nonempty $\varepsilon$-cores (Theorem 1 below).

Let the cooperative game $v$ with the set $N$ of players be given by its characteristic function

$$
v: 2^{N} \rightarrow \mathbb{R},
$$

where $v(\phi)=0$. Denoting by $1_{s}$ the indicator function of an arbitrary coalition $S \subseteq N$, we say that $v$ is $\varepsilon$-balanced for $\varepsilon \geq 0$ if for all $S_{1}, S_{2}, \ldots, S_{k} \subset N$ and parameters $\lambda\left(S_{1}\right), \ldots, \lambda\left(S_{k}\right) \in \mathbb{R}_{+}$,

$$
\sum_{j=1}^{k} \lambda\left(S_{j}\right) 1_{S_{j}}=1_{N} \quad \text { implies }(1-\varepsilon) \sum_{j=1}^{k} \lambda\left(S_{j}\right) v\left(S_{j}\right) \leq v(N)
$$

The $\varepsilon$-core $K_{\varepsilon}(v)$ of $v$ consists of all allocation vectors $x \in \mathbb{R}^{N}$ such that

i) $x(N)=v(N)$

ii) $x(S) \geq(1-\varepsilon) v(S)$ for all $S \subset N$,

where, as usual, $x(S)=\sum_{i \in S} x(i)$. 
Our first result seemingly extends the theorem of Bondareva and Shapley (the case $\varepsilon=0$ in our notation). Actually, it is easily seen to be equivalent to that theorem by considering the game $v_{\varepsilon}: 2^{N} \rightarrow \mathbb{R}$, where

$$
v_{\varepsilon}(S)= \begin{cases}(1-\varepsilon) v(S) & \text { if } S \neq N \\ v(S) & \text { if } S=N\end{cases}
$$

We therefore only sketch the simple direct proof.

Theorem 1: The game $v: 2^{N} \rightarrow \mathbb{R}$ is $\varepsilon$-balanced if and only if $K_{\varepsilon}(v) \neq \phi$.

Proof: Consider the linear program (LP):

$$
\max \lambda_{N} v(N)+(1-\varepsilon) \sum_{S \subset N} \lambda_{S} v(S)
$$

such that $\lambda_{N}+\sum_{S \ni i} \lambda_{S} \leq 0$

$$
\lambda_{N} \text { free, } \quad \lambda_{s} \geq 0(S \subset N) .
$$

By linear programming duality, $K_{\varepsilon}(v) \neq \phi$ is tantamount to saying that each feasible solution $\lambda$ for (LP) yields objective function value $\leq 0$, i.e., any choice of numbers $\lambda_{S}^{\prime} \geq 0(S \subset N)$ and $\lambda_{N}^{\prime}$ with the property

$$
\sum_{S \ni i} \lambda_{S}^{\prime}=\lambda_{N}^{\prime} \quad \text { for all } i \in N
$$

necessarily satisfies $(1-\varepsilon) \sum_{S \in N} \lambda_{S}^{\prime} v(S) \leq \lambda_{N}^{\prime} v(N)$

Rather than verifying the condition for $\varepsilon$-balancedness directly, Theorem 1 allows us to exhibit allocation vectors in the $\varepsilon$-core instead. We will now turn our attention to the study of $\varepsilon$-balancedness of some combinatorial games that are known to be generally not $O$-balanced.

Before doing so, we want to relate our $\varepsilon$-core with the $\varepsilon$-tax core of Tijs and Driessen [1986] that consists of those vectors $x \in \mathbb{R}^{N}$ with the properties

i) $x(N)=v(N)$

ii) $x(S) \geq(1-\varepsilon) v(S)+\varepsilon \sum_{i \in S} v(i) \quad$ if $S \subset N$. 
It is not hard to find games with empty $\varepsilon$-tax core but non-empty $\varepsilon$-core. Indeed, for non-negative games, the $\varepsilon$-core is a more general solution concept. On the other hand, both concepts coincide for games $v$ with $v(i)=0$ for each $i \in N$. Examples of the latter are the matching games in Section 3.

A vector $x \in \mathbb{R}^{N}$ is an imputation for the game $v$ is $x$ distributes $v(N)$ and is individually rational, i.e., if

i) $x(N)=v(N)$

ii') $x(i) \geq v(i)$ for all $i \in N$.

Note that any vector in the $\varepsilon$-tax core is an imputation while the $\varepsilon$-core may contain also vectors that are not individually rational. In the games we consider below, however, we will always exhibit imputations in the $\varepsilon$-core.

\section{Matching Games}

Let $G=(N, E)$ be a graph on the vertex set $N$ with the set $E$ of edges. We assume that $G$ is (edge-) weighted via

$w: E \rightarrow \mathbb{R}$.

Thinking of $N$ as a set of players, define the value $v(S)$ of the coalition $S \subseteq N$ in the matching game $v$ relative to $w$ by

$$
v(S)=\max \{w(M) \mid M \subseteq E(S) \text { matching }\},
$$

where $E(S)$ is the set of edges joining two vertices in $S$. (Recall that a matching in $G$ is a set of pairwise disjoint edges).

It is well-known that the matching game is $O$-balanced if the graph $G$ is bipartite, i.e., if $G$ contains no circuit of odd length $\geq 3$ (cf. Shapley and Shubik [1972]). On the other hand, there are matching games with empty $O$-core (take $G$ to be triangle with $w \equiv 1$ ).

Theorem 2: Assume that $G=(N, E)$ contains no circuit of odd length smaller than $k$. Then the associated matching game $v$ is $\frac{1}{k}$-balanced. 
Proof: For any coalition $S \subseteq N, v(S)$ is the optimal value of the integer linear program $I L P(S)$ :

$$
\max \sum_{e \in E} w(e) x_{e}
$$

such that $\sum_{e \ni i} x_{e} \leq 1 \quad$ if $i \in S$

$$
\begin{gathered}
\sum_{e \ni i} x_{e} \leq 0 \quad \text { if } i \notin S \\
x_{e} \in\{0,1\} .
\end{gathered}
$$

Relaxing the integrality constraints $x_{e} \in\{0,1\}$ to $0 \leq x_{e} \leq 1$, we obtain the associated linear program $L P(S)$, whose optimal value we denote by $\bar{v}(S)$. Clearly $\bar{v}(S) \geq v(S)$.

The game $\bar{v}$ is a linear production game in the sense of Owen [1975] and hence has a non-empty $O$-core. Choose $\bar{z} \in K_{0}(\bar{v})$ and define $z \in \mathbb{R}^{N}$ via

$$
z(i)=\bar{z}(i) \cdot \frac{v(N)}{\bar{v}(N)} \quad \text { for all } i \in N
$$

By Lemma 3 below, we have in particular $v(N) / \bar{v}(N) \geq\left(1-\frac{1}{k}\right)$. Thus

$$
\begin{aligned}
z(S) & =\bar{z}(S) \cdot \frac{v(N)}{\bar{v}(N)} \geq \bar{v}(S) \cdot \frac{v(N)}{\bar{v}(N)} \\
& \geq v(S) \cdot\left(1-\frac{1}{k}\right)
\end{aligned}
$$

Lemma 3: Under the conditions of the proof of Theorem 2, we have $v(S) \geq\left(1-\frac{1}{k}\right) \bar{v}(S)$.

Proof: We use the fact (cf. Lovász and Plummer [1986], Chap. 7) that the linear program $L P(S)$ has an optimal solution $x \in \mathbb{R}^{E}$ such that for all $e \in E$,

$$
x_{e}=0 \quad \text { or } \quad x_{e}=\frac{1}{2} \quad \text { or } \quad x_{e}=1 .
$$


Moreover, the set $\left\{e \in E \mid x_{e}=\frac{1}{2}\right\}$ is a union of a disjoint circuits of odd length. Let $C$ be such a circuit. The contribution of $C$ towards $\bar{v}(S)$ is

$$
\frac{1}{2} \cdot \sum_{e \in C} w(e)
$$

We claim that $C$ contains a matching $M$ of weight

$$
w(M) \geq\left(1-\frac{1}{k}\right) \cdot \frac{1}{2} \sum_{e \in C} w(e)
$$

Let the edge $m \in C$ have minimal weight in $C$. Now $C \backslash\{m\}$ contains two disjoint matchings because $|C|$ is odd. So one of them has weight at least

$$
\begin{aligned}
\frac{1}{2}\left[\sum_{e \in C} w(e)-w(m)\right] & \geq \frac{1}{2} \sum_{e \in C} w(e)-\frac{1}{2} \frac{1}{|C|} \sum_{e \in C} w(e) \\
& \geq\left(1-\frac{1}{k}\right) \cdot \frac{1}{2} \sum_{e \in C} w(e) .
\end{aligned}
$$

Thus, by replacing all odd circuits $C$ with $x_{\mid C} \equiv \frac{1}{2}$ by an appropriate matching, we obtain a matching in $S$ with weight at least $\left(1-\frac{1}{k}\right) \bar{v}(S)$.

We remark that Theorem 2 is "best possible". It is straightforward to see that the cardinality matching game (i.e., $w \equiv 1$ ) on an odd circuit of length $k$ is not $\varepsilon$-balanced for any $\varepsilon<\frac{1}{k}$.

Corollary 4: (cf. Shapley and Shubik [1972]): If $G$ is bipartite, then the matching game is balanced.

\section{Bin Packing Games}

We introduce the following combinatorial game: There are $n$ items of sizes $a_{1}$, $\ldots, a_{n} \in \mathbb{R}_{+}$and $m$ bins of sizes $b_{1}, \ldots, b_{m} \in \mathbb{R}$, where we assume that each item $i$ fits into any bin $j$, i.e., $a_{i} \leq b_{i}$ for all $i$ and all $j$. The set $N$ of players consists of 
all items and all bins. In particular, $|N|=n+m$. A coalition $S \subseteq N$ comprises a set $A \subseteq\{1, \ldots, n\}$ of items and a set $B \subseteq\{1, \ldots, m\}$ of bins. The value $v(S)$ is defined as the maximum total size of items in $A$ that can be packed into bins in $B$. So

$$
v(S)=\max \sum_{j=1}^{|B|} \sum_{i \in A_{j}} a_{i}
$$

where the maximum is taken over all pairwise disjoint subsets $A_{i}, \ldots, A_{|B|}$ of $A$ such that

$$
\sum_{i \in A_{j}} a_{i} \leq b_{j}
$$

To illustrate a bin packing game, imagine that the "bins" are trucks of capacity $b_{j}$. The items produce a profit proportional to their size $a_{i}$ if they are brought to a market place. The value $v(N)$ of the grand coalition thus represents the maximum profit achievable. Note that $v(N)$ is bounded by $\min \left\{\sum a_{i}, \sum b_{j}\right\}$.

How should $v(N)$ be allocated to the "owners" of the items and the "owners" of the bins?

We first show that we cannot guarantee $\varepsilon$-balancedness for general bin packing games unless $\varepsilon \geq \frac{1}{7}$.

Proposition 5: Let $\varepsilon \geq 0$ be such that every bin packing game is $\varepsilon$-balanced. Then $\varepsilon \geq \frac{1}{7}$.

Proof: Take 2 bins with capacity $b_{1}=b_{1}=2$ and 5 items of size $a_{1}=a_{2}=$ $1+\delta, a_{1}^{\prime}=a_{2}^{\prime}=a_{3}^{\prime}=1$ for some $0<\delta<1$.

Consider the coalitions $S_{1}=\left\{b_{1}, a_{1}^{\prime}, a_{2}^{\prime}\right\}, S_{2}=\left\{b_{2}, a_{2}^{\prime}, a_{3}^{\prime}\right\}, S_{3}=\left\{b_{1}, b_{2}, a_{1}\right.$, $\left.a_{2}, a_{1}^{\prime}, a_{3}^{\prime}\right\}$ and $S_{4}=\left\{a_{1}, a_{2}\right\}$.

Then $\sum_{j=1}^{4} 1_{s_{j}}=2 \cdot 1_{N}$ and, therefore, the balancedness conditions imply

$$
(1-\varepsilon) \sum_{j=1}^{4} v\left(S_{j}\right) \leq 2 \cdot v(N)=6+2 \delta
$$

Now $v\left(S_{1}\right)=v\left(S_{2}\right)=2, v\left(S_{3}\right)=3+\delta, v\left(S_{4}\right)=0$, Letting $\delta \rightarrow 0$, we see that $\varepsilon \geq \frac{1}{7}$ must hold. 
Theorem 6: Each bin packing game is $\frac{1}{2}$-balanced.

Proof: For the items $a_{1}, \ldots, a_{n}$ and bins $b_{1}, \ldots, b_{m}$, we set $a=a_{1}+\cdots+a_{n}$ and $b=b_{1}+\cdots+b_{m}$. There are two cases to be distinguished.

Case 1: $a>v(N)$ (i.e. the grand coalition is short of truck drivers).

We allocate nothing to the item owners and assign to truck driver $j$ the value

$$
x_{j}=\frac{b_{j}}{b} v(N) .
$$

We must show that $x(S) \geq \frac{1}{2} v(S)$ holds for any coalition $S$ in the bin packing game. This will follow once we can demonstrate that there is an optimal bin packing in which every bin is at least half full because then $v(N) \geq \frac{1}{2} b$ and so $x(S) \geq \frac{1}{2} \sum_{j \in S} b_{j} \geq \frac{1}{2} v(S)$.

Consider an optimal bin packing and suppose bin $j$ is less than half full. Because $a>v(N)$, there is an item $a_{i}$ that is not in any bin. Because the current packing is optimal, we have $a_{i} \geq \frac{1}{2} b_{j}$. On the other hand, $a_{i} \leq b_{j}$ (the fundamental assumption in our model) implies that we would improve the current solution by replacing the content of bin $j$ with $a_{i}$.

Case 2: $a=v(N)$.

In this case, we assign nothing to the truck owners and allocate to the owner of item $i$ the value

$$
x_{i}=a_{i} .
$$

This allocation obviously satisfies $x(S) \geq v(S)$ for any coalition $S$.

Our results show that the smallest $\varepsilon$ for which every bin packing game is $\varepsilon$-balanced lies between $1 / 7$ and $1 / 2$. Are there sharper bounds? It should also be interesting to study the bin packing game in a more general framework where $a_{i} \leq b_{j}$ does not necessarily hold and the profit $p_{i}$ gained from packing item $a_{i}$ may be independent from its size. 


\section{TSP Games}

TSP games ("Traveling Salesman" games) can be motivated by a repairman who starts from his home 0 and visits the customers $N=\{1, \ldots, n\}$ before returning home (cf. Tamir [1989] and Potters et al. [1992]). Hence we consider a graph $G$ on the vertex set $N \cup\{0\}$ whose edges correspond to links connecting customers. Each link $e$ is weighted by its (non-negative) length $d(e)$. Taking $N$ to be a set of players, the associated TSP game evaluates the cost $c(S)$ of any coalition $S \subseteq N$ as

$c(S)=$ length of a shortest tour through $S \cup\{0\}$.

We seek to distribute the total cost $c(N)$ of the grand coalition among the players $N$. Similarly to the foregoing, we define the $\varepsilon$-core $K_{\varepsilon}(c)$ of the cost game $c$ to consist of all vectors $x \in \mathbb{R}^{N}$ such that

i) $x(N)=c(N)$

ii) $x(S) \leq(1+\varepsilon) c(S)$ for all $S \subset N$.

In the model we discuss in this section, we will assume throughout that the graph $G$ is undirected and that the edge lengths satisfy the triangle inequality. Without going into details, let us remark here that one can exhibit an example of a TSP game with empty $O$-core whose underlying graph satisfies the above requirements (Tamir [1989]).

In fact, there are even euclidean TSP games with empty $O$-core (Faigle and Kern [1993]). (Recall that a euclidean TSP game, by definition, arises from choosing the vertex set in the plane $\mathbb{R}^{n}$ and considering the euclidean distances between pairs of points (see also below)).

In the following, we denote by $L=v(N)$ the length of the shortest tour and by $T$ the length of a minimum spanning tree in $G$.

Theorem 7: If $L \leq(1+\varepsilon) T$, then the TSP game $c$ has a non-empty $\varepsilon$-core.

Proof: The MST ("Minimum Spanning Tree") game $\bar{c}$ associated with $G$ is given by

$$
\bar{c}(S)=\text { length of an MST on } S \cup\{0\} .
$$

It is well-known that $\bar{c}$ has a non-empty $O$-core (cf. Claus and Kleitman [1973]). 
Choose $\bar{x} \in K_{0}(\bar{c})$ and define $x \in \mathbb{R}^{N}$ via

$$
x(i)=\bar{x}(i) \frac{L}{T} \quad \text { for } i \in N
$$

We claim: $x \in K_{\varepsilon}(c)$.

Because $T=\bar{x}(N)$, we obtain $x(N)=L$. Moreover, $c(S) \geq \bar{c}(S)$ implies

$$
x(S)=\bar{x}(S) \frac{L}{T} \leq \bar{c}(S)(1+\varepsilon) \leq(1+\varepsilon) c(S) .
$$

Note that $L \leq 2 T$ always holds since a (in general non-optimal) tour can be constructed by the following standard procedure: double the edges of the optimal tree in order to obtain an eulerian graph on $N \cup\{0\}$. Trace the eulerian tour of length $2 T$ and take "shortcuts" using the triangle inequality. There results a tour of length $\leq 2 T$.

In many situations, the $\varepsilon$ in the hypothesis of Theorem 6 is quite small. Consider, for example, the euclidean TSP and let $U:=[0,1]^{2}$ be the unit square with center $x_{0}=\left(\frac{1}{2}, \frac{1}{2}\right) \in U$. Choose $x_{1}, \ldots, x_{n} \in U$ and define $G=(V, E)$ to be the complete graph on $V=N \cup\{0\}$ with edge lengths

$$
c(i, j)=\left\|x_{i}-x_{j}\right\|_{2}
$$

It is known that with high probability

$$
L \approx 0.72 \sqrt{n} \text { and } T \approx 0.68 \sqrt{n}
$$

(cf. Kern [1989], Rhee and Talagrand [1989], Steele [1990], Goemans and Bertsimas [1991]). More precisely, it has been shown that there exist constants $\beta_{L}$ and $\beta_{T}$ such that for independent, uniformly distributed random variables $X_{1}, X_{2}, \ldots$ in $U$,

$$
\operatorname{Prob}\left(\left|L\left(X_{1}, \ldots, X_{n}\right)-\beta_{L} \sqrt{n}\right| \geq \alpha \sqrt{n}\right)
$$

and

$$
\operatorname{Prob}\left(\left|T\left(X_{1}, \ldots, X_{n}\right)-\beta_{T} \sqrt{n}\right| \geq \alpha \sqrt{n}\right)
$$

become exponentially small as $n \rightarrow \infty$ for any fixed $\alpha>0$. Numerical experiments (Goemans and Bertsimas [1991]) suggest that $\beta_{L} \approx 0.72$ and $\beta_{T} \approx 0.68$. 
Hence, for large $n$, we will have

$$
L \leq 1.06 T
$$

with high probability, which guarantees an $\varepsilon$-core with $\varepsilon \approx 0.06$.

Another possible allocation rule for the TSP game is motivated by the following relaxation of the TSP problem. As above, let $c_{i j}$ denote the distance between nodes $i$ and $j$. Define, for each coalition $S \subseteq N$

$$
\begin{aligned}
\tilde{c}(S):= & \min \sum_{(i, j) \in E} c_{i j} x_{i j} \\
& \sum_{(i, j) \in E} x_{i j}= \begin{cases}2 & \forall i \in S \cup\{0\} \\
0 & \text { else }\end{cases} \\
& \sum_{i \in T, i \notin T} x_{i j} \geq 2 \quad \forall \varnothing \subset T \subset S \cup\{0\} \\
& x_{i j} \geq 0 .
\end{aligned}
$$

Obviously, any optimal tour through $S \cup\{0\}$ satisfies these constraints (so called "degree constraints" and "subtour elimination constraints"). As observed already by Tamir [1989], [1991] and others, the game defined by $\tilde{c}$ has nonempty core. (In fact, a vector $\tilde{x}$ in the core of this game can be computed efficiently.) If $\tilde{x}$ is a vector in the core of the game defined by $\tilde{c}$, then

$$
x(i):=\tilde{x}(i) \cdot \frac{L}{\tilde{c}(N)},
$$

(where, again, $L$ denotes the length of an optimum tour through $N \cup\{0\}$ ) defines an allocation for the TSP game. Obviously,

$$
x(S) \leq \tilde{x}(S) \cdot \frac{L}{\tilde{c}(N)} \leq \tilde{c}(S) \cdot \frac{L}{\tilde{c}(N)} \leq c(S) \cdot \frac{1}{\tilde{c}(N)} .
$$

It has been shown by Wolsey [1980] that the ratio $L / \tilde{c}(N)$ is at most $3 / 2$, assuming the triangle inequality for the distances $c_{i j}$. Thus, this allocation rule yields a vector $x$ in the $\frac{1}{2}$-core of the TSP-game. It has been conjectured that the above ratio is bounded by $4 / 3$. In the euclidean case, empirical results on random problems with uniformly distributed points in the unit sqare seem to imply 
that, on the average,

$$
c(N) \geq 0.70 \cdot \sqrt{n}
$$

(cf. Goemans and Bertsimas [1991]). Thus, with high probability, a random TSP game has nonempty $\varepsilon$-core even for

$$
\varepsilon \approx \frac{0.72}{0.70} \approx 0.03
$$

\section{References}

Bondareva ON (1963) Some applications of linear programming methods to the theory of cooperative games. Problemy Kibernet 10:119-139 (in Russian)

Claus A, Kleitman DJ (1973) Cost allocation for a spanning tree. Networks 3:289-304

Faigle U, Kern W (1993) unpublished

Goemans M, Bertsimas D (1991) Probabilistic analysis of the Held and Karp lower bound for the euclidean TSP. Math of OR 16:72-89

Kern W (1989) On the rate of convergence of some stochastic processes. Math of OR 14:275-280

Lovász L, Plummer MD (1986) Matching theory. North Holland Math Studies 121, North Holland, Amsterdam

Owen G (1975) The core of linear production games. Math Programming 9:358-370

Potters JAM, Curiel IJ, Tijs SH (1992) Traveling salesman games. Math Progr 53:199 211

Rhee WT, Talagrand M (1989) A sharp deviation for the stochastic TSP. Ann Probab 17:1-8

Shapley LS (1967) On balanced sets and cores. Naval Res Logist Quaterly 14:453-460

Shapley LS, Shubik M (1966) Quasi-cores in a monetary economy with nonconvex preferences. Econometrica 34:805-827

Shapley LS, Shubik M (1972) The assignment game I: The core. Intern J Game Theory 1:111-130

Steele M (1990) Probabilistic and worst case analyses of classical problems of combinatorial optimization in euclidean space. Math of OR 15:749-770

Tamir A (1989) On the core of a traveling salesman cost allocation game. OR Letters 8:31-34

Tamir A (1991) On the core of network synthesis games. Math Programming 50:123-135

Tijs SH, Driessen TSH (1986) Extensions of solution concepts by means of multiplicative $\varepsilon$-tax games. Math Social Sciences 12:9-20

Wolsey LA (1980) Heuristic analysis, Linear programming and branch and bound. Math Programming Study 13:121-134 